\title{
Assessment of Microcirculation in the Diabetic Foot with Laser Speckle Contrast Imaging
}

Category: Original article

Running head: LSCI MICROCIRCULATION ASSESSMENT IN DIABETIC FEET

O.A. Mennes, ${ }^{\text {a,b, }}$ J.J. van Netten, ${ }^{\text {a,c,d }}$ J.G. van Baal, ${ }^{\text {a,e }}$ W. Steenbergen ${ }^{\text {b }}$

a Ziekenhuisgroep Twente, Department of vascular surgery, Zilvermeeuw 1, Almelo, The Netherlands

${ }^{\mathrm{b}}$ University of Twente, Faculty of Science and Technology, Biomedical Photonic Imaging, Drienerlolaan 5, Enschede, The Netherlands

c Queensland University of Technology, School of Clinical Sciences, Institute of Health and Biomedical Innovation, 2 George St, Brisbane City, Australia

d Amsterdam UMC, location AMC, Department of Rehabilitation, Amsterdam Movement Sciences, Meibergdreef 9, Amsterdam, the NetherlanFds

${ }^{\mathrm{e}}$ Cardiff University, School of Medicine, UHW Main Building, Health Park, Cardiff, CF14 4XN, Wales, United Kingdom

\section{*Corresponding author:}

Onno August Mennes,

O.A. Mennes, Department of Surgery

Address: Zilvermeeuw 1; Postbus 7600; 7600 SZ Almelo

Phone +31 88708 3727; Fax +31 53 4891105; E-mail o.mennes@ zgt.nl

Manuscript keywords: Laser Speckle Contrast Imaging, Diabetes Mellitus, Diabetes Complications, Foot Ulcer, Microcirculation, Peripheral Artery Disease,

Wordcount: 3641 


\begin{abstract}
Objective A major challenge for treating diabetic foot ulcers is estimating the severity of ischemia, as currently used non-invasive diagnostic techniques provide relatively poor prognostic values. Laser Speckle Contrast Imaging (LSCI) is a promising non-invasive technique to assess microcirculation. Our aim was to investigate the stability and reproducibility of LSCI for the assessment of microcirculation in the diabetic foot, the relation of LSCI results to currently used non-invasive blood pressure measurements, and the ability of LSCI to discriminate between the degrees of ischemia.
\end{abstract}

Approach Thirty-three participants with diabetic foot ulcers were included in this prospective, single centre, observational cohort study that was conducted in the Netherlands. They were classified as non-ischemic, ischemic or critical-ischemic based on criteria formulated in the international guidelines. Two clinicians performed LSCI scans of the foot, consisting of baseline measurements, followed by two stress tests (post-occlusion peak and elevation test). With 3 measurement conditions and 5 regions of interest of the foot per patient, a total of 15 measurements were available for analyses.

Main results The intra-observer agreement of LSCI was high (Interclass Correlation Coefficient $(\mathrm{ICC})=0.711-0.950 ; p<0.001$ ) for all 15 measurements. The inter-observer agreement was high $(\mathrm{ICC}=0.728-0.861 ; p \leq 0.001)$ for 10 measurements and moderate (ICC $=0.476-0.570 ; p \leq 0.005)$ for the remaining 5 measurements. The inter-assessor agreement was high and significant ( $\mathrm{ICC}=0.857-0.996 ; \mathrm{p} \leq 0.001)$ for all measurements. Correlation between LSCI and non-invasive blood pressure measurements was low (ICC $=-0.272-0.582$ ). During both stress tests, microcirculation was significantly lower in critical-ischemic feet compared to non-ischemic feet (67.5 Perfusion Units (PU) vs. 96.3PU and 41.0PU vs. 63.9PU; $\mathrm{p}<0.05)$.

Significance LSCI is a stable and reproducible technique for assessment of microcirculation in people with diabetic foot ulcers and shows significant differences between non-ischemic, ischemic and critical-ischemic patient populations.

Acknowledgements: This work is supported by NWO-TTW (project number 14538), Stichting Diavasc and Ziekenhuisgroep Twente.

Author Disclosure Statement: Perimed AB (Stockholm, Sweden) provided the equipment used in this study, but had no further involvement in the research or interpretation of the results of this study. 


\section{Introduction}

One of the major complications of diabetes mellitus are diabetic foot ulcers, with an annual prevalence around 2\% and a life-time incidence between $19 \%$ and 34\% (1-4). Diabetic foot ulcers have a high morbidity and mortality, resulting in increasing healthcare costs (5-8) and are an important reason to perform lower extremity amputation, especially in the presence of ischemia (5).

Early and accurate diagnosis is the first step in ischemia treatment and outcome improvement (2). Primary diagnosis of ischemia currently consists of visual inspection and noninvasive assessment of blood pressure in the feet (2). Clinical decisions are based on these measurements; according to international guidelines, urgent vascular imaging and revascularisation are to be considered in patients with a diabetic foot ulcer and either an ankle pressure $<50 \mathrm{mmHg}$ or an ankle-brachial index $(\mathrm{ABI})<0.5$, in patients with a toe pressure $<30$ $\mathrm{mmHg}$, or in patients with a transcutaneous oxygen pressure $\left(\mathrm{Tc} p \mathrm{O}_{2}\right)<25 \mathrm{mmHg}$ (2).

However, these techniques only provide relatively poor indications of the severity of ischemia. Furthermore, these values provide relatively poor prognostic values in predicting ulcer healing (9). A suggested explanation is that these measurements are not necessarily reflecting the situation of ischemia at the site of the ulcer. With different angiosomes providing blood supply to the feet, low values in one angiosome may not reflect blood supply at the ulcer location (10). Clinical evidence adds to the poor prognostic values; for example, a large observational cohort study showed some patients with critical ischemia to heal from their foot ulcers without vascular interventions, while some patients with non-ischemic ulcers did not heal despite adequate treatment (11).

Improving assessment of microcirculation in people with diabetic foot ulcers is an important area for research, as identified in international guidelines (2). This may overcome the disadvantages of currently used non-invasive blood pressure measurements (2). An improved diagnostic technique should reflect the situation of ischemia at the ulcer location, be stable (i.e. provide a constant signal over time during the measurements) and reproducible (i.e. have high intra and inter-user correlation), non-invasive, and differ from currently used non-invasive blood pressure measurements.

A potential technique fulfilling these requirements is single-exposure Laser Speckle Contrast Imaging (LSCI). LSCI can measure microcirculation based on changes in the optical speckle pattern generated by motion of red blood cells in the skin or ulcers (12). When skin is illuminated with coherent laser light, the reflected light will create light and dark areas, a socalled speckle pattern (12). In perfused skin, moving red blood cells cause a dynamic speckle 
pattern. The dynamic character of the speckles can be visualized with a camera with an integration time longer than the time scale of the speckle fluctuations, causing the speckle pattern to be blurred on the recorded image (12-14). Increased perfusion (i.e. increased local concentration and/or increased speed of moving red blood cells), result in a reduced spatial speckle contrast $(12,15)$. There is a correlation between the speckle contrast and blood perfusion. By measuring this contrast, LSCI can be used to estimate blood perfusion in the capillary bed at the exact location of the ulcer $(12,14)$.

LSCI is already successfully applied in the assessment of burns and burn-related wounds (16). In people with diabetes, it has been used to assess the microvascular endothelial function of the arm in a population with type 1 diabetes (17). A stress test (post-occlusion peak test) was performed to differentiate between people with diabetes and a control group. Furthermore, a conference paper has reported a pilot study testing LSCI on the feet of 5 patients (3 with diabetes) (18). However, no additional clinical baseline information was provided and only 4 diabetic foot ulcers were measured in the 3 patients. Both studies concluded that LSCI could be used for diagnostic screening or monitoring of the capillary blood flow on a regular basis during treatment $(17,18)$. However, neither of these studies investigated whether LSCI is a method that can be reliably used in people with diabetes. Its stability, reproducibility, reliability and validity have not yet been investigated.

Our aim is to investigate the stability and reproducibility of LSCI for the assessment of microcirculation in the diabetic foot and its relation to currently used non-invasive blood pressure measurements and stress tests.

\section{Methods}

For this prospective, single centre, observational cohort study, 33 consecutive participants with diabetic foot ulcers were recruited from the 'diabetic foot outpatient clinic' in Ziekenhuisgroep (Hospital Group) Twente, location Almelo, the Netherlands. Sample size calculation was not applicable, as no baseline information for LSCI in people with diabetic foot ulcers was available. All participants had diabetes mellitus type I or II, were 18 years or older and were able to undergo non-invasive blood pressure measurements. Participants with an ulcer on both feet or with a foot infection (International Working Group on the Diabetic Foot grade 2-4 (19)) were excluded. The study protocol was approved by the medical research ethics committee Twente (NL52422.044.15), and was registered in the Dutch Trial Register (NTR5116). All participants gave written informed consent before participation in the study. 
All participants underwent non-invasive blood pressure measurements consisting of brachial pressure, ankle pressure, toe pressure (measured at the hallux, or, in case the hallux was amputated, at the second toe), and transcutaneous oxygen pressure $\left(\operatorname{Tc} p \mathrm{O}_{2}\right)$ measurements (Periflux 6000, Perimed AB, Stockholm, Sweden); ankle-brachial index (ABI) and toe-brachial index (TBI) were calculated. Participants were classified as non-ischemic, ischemic or criticalischemic based on the lowest measured blood pressure using cut-off points based on international guidelines (2,20): participants with an ankle pressure $<50 \mathrm{mmHg}, \mathrm{ABI}<0.5$, toe pressure $<30 \mathrm{mmHg}$, TBI $<0.3$, or $\mathrm{Tc} p \mathrm{O}_{2}<25 \mathrm{mmHg}$ were classified as 'critical-ischemic'; participants with an ankle pressure $50-70 \mathrm{mmHg}$, ABI 0.5-0.9, toe pressure $30-50 \mathrm{mmHg}$, TBI 0.3-0.7 or $\mathrm{Tc}_{\mathrm{p}} \mathrm{O}_{2}$ 25-30 mmHg were classified as 'ischemic'; non-ischemic participants had values above those cut-off points, i.e.: ankle pressure $>70 \mathrm{mmHg}, \mathrm{ABI}>0.9$, toe pressure $>50$ $\mathrm{mmHg}$, TBI $>0.7$, and $\mathrm{Tc} p \mathrm{O}_{2}>30 \mathrm{mmHg}(2,20)$.

Following blood pressure measurements, multiple LSCI scans were performed in each participant (PeriCam PSI; Perimed AB, Stockholm, Sweden). Before use the system was calibrated with its calibration box, measuring a zero-perfusion area and a colloidal suspension of polystyrene particles to set the LSCI values on $0 \pm 5$ perfusion units (PU) and $250 \pm 5 \mathrm{PU}$ respectively. Both the dorsal and plantar side of the foot were scanned, but only the ulcerated side was used in data analysis. The scans were made with approximately $25 \mathrm{~cm}$ distance between the system and the foot, a framerate of $10 \mathrm{fps}$, integration time of $6 \mathrm{~ms}$, a resolution of $1388 \times 1038$ pixels and a measurement area of $25 \times 15 \mathrm{~cm}$. The ulcerated foot was scanned twice by the principal investigator to calculate intra-observer variability. For 25 participants, a complete additional LSCI scan of the ulcer was performed by a trained health professional (registered physician assistant with $>10$ years of experience in treating diabetic foot disease) as second observer to calculate inter-observer variability.

Both the non-invasive blood pressure measurement and the LSCI scans were performed in a temperature controlled hospital room $\left(21 \pm 1^{\circ} \mathrm{C}\right)$. All patients underwent wound treatment before the perfusion measurements took place, during which procedure the foot was able to acclimatize to this room temperature. The LSCI scan methodology is schematically shown in Fig. 1. Baseline microcirculation was determined in the first period of the scan, and defined as 'no visual changes in the mean LSCI signal value for 30 seconds'. Subsequently, two stress tests (post-occlusion peak and elevation test, as described below) were performed, in order to increase a potential difference between patient populations and to assess microcirculation and endothelial function in more detail. Between and after those stress tests an extra baseline measurement of 30 seconds was performed to monitor the stability of LSCI over time. 

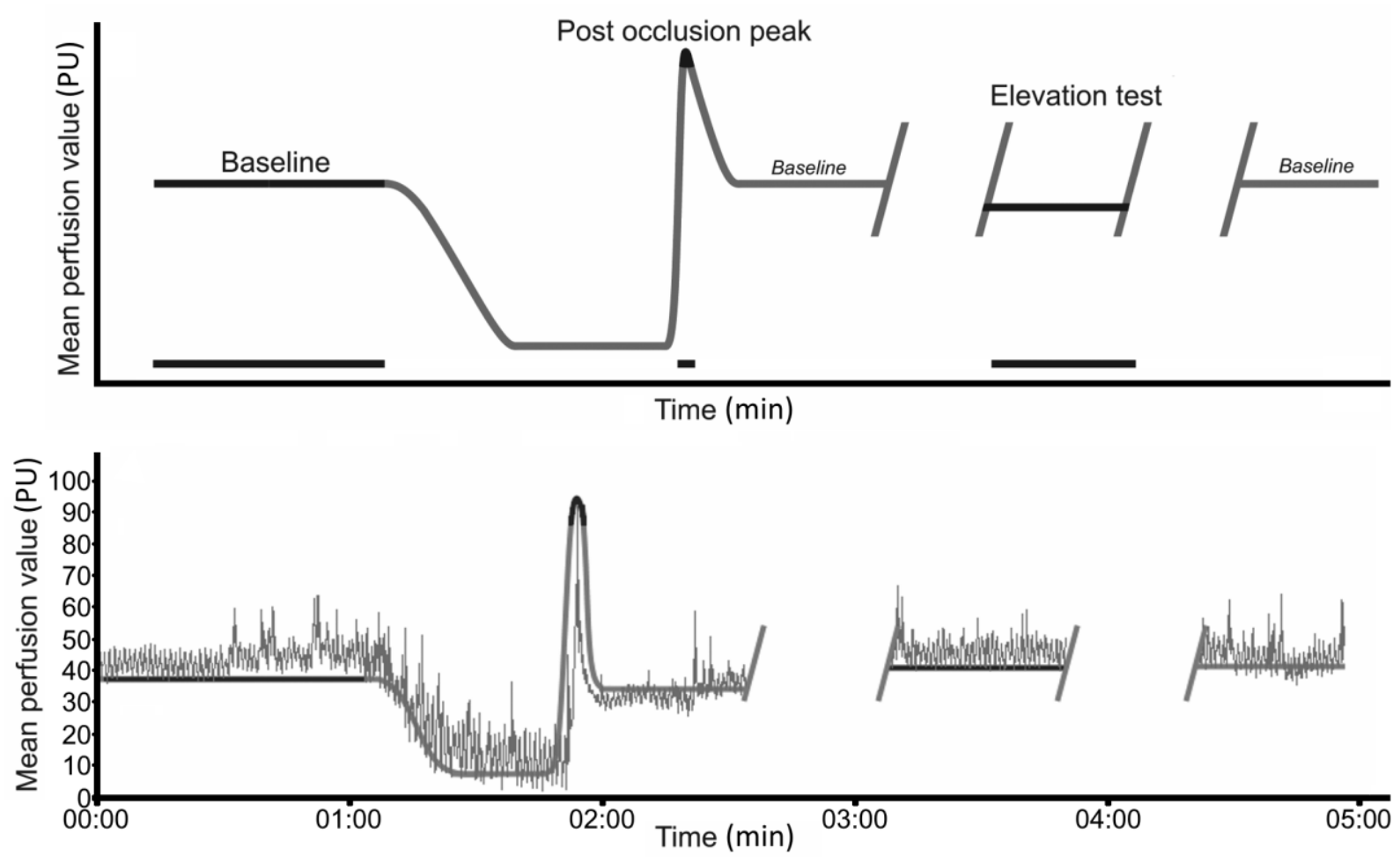

Fig. 1 Top: Schematic overview of different stress tests and Laser Speckle Contrast Imaging signal over time with measurement periods shown in black. Baselines and elevation test all had a minimum duration of 30 seconds. Bottom: Example of the outcomes of the LSCI scan in one participant, with mean perfusion values for each ROI over time.

During the post-occlusion peak test, blood flow in the foot was interrupted with a pressure cuff (max $260 \mathrm{mmHg}$ ) around the ankle. The pressure was released after 30 seconds. The maximum perfusion value during the post-occlusive reactive hyperaemia was measured as outcome. During the elevation test, the foot was lifted on a foam block of $30 \mathrm{~cm}$ height (with the scan paused during placement). Elevation test measurements consisted of a minimum of 30 seconds of a stable signal in the elevated foot, with its mean value as outcome.

The scans were quantified by measuring the perfusion of five specified Regions Of Interest (ROI), referring to a physical position on the image: ulcer (ulcer location); ulcer edge (ulcer edges and surrounding $1 \mathrm{~cm}$ ); $\mathrm{Tc}_{\mathrm{p}} \mathrm{O}_{2}$ (probe measurement area); hallux (total visible hallux, or second toe when hallux was amputated); foot (total visible foot) - see Fig. 2. All ROIs were drawn on the scan by the principal investigator using LSCI-software (PIMSoft version 1.5; Perimed AB, Stockholm Sweden). Positions of the ROI were adjusted in each timeframe by the principal investigator to correct for motion effects. In order to measure the influence of the different assessors on the rating of the LSCI scans, for the same 25 participants that received an extra scan from the trained health professional an extra analysis was performed 
by an independent researcher who was not involved in the LSCI scans. This researcher quantified the scan from the trained health professional via the method described above.
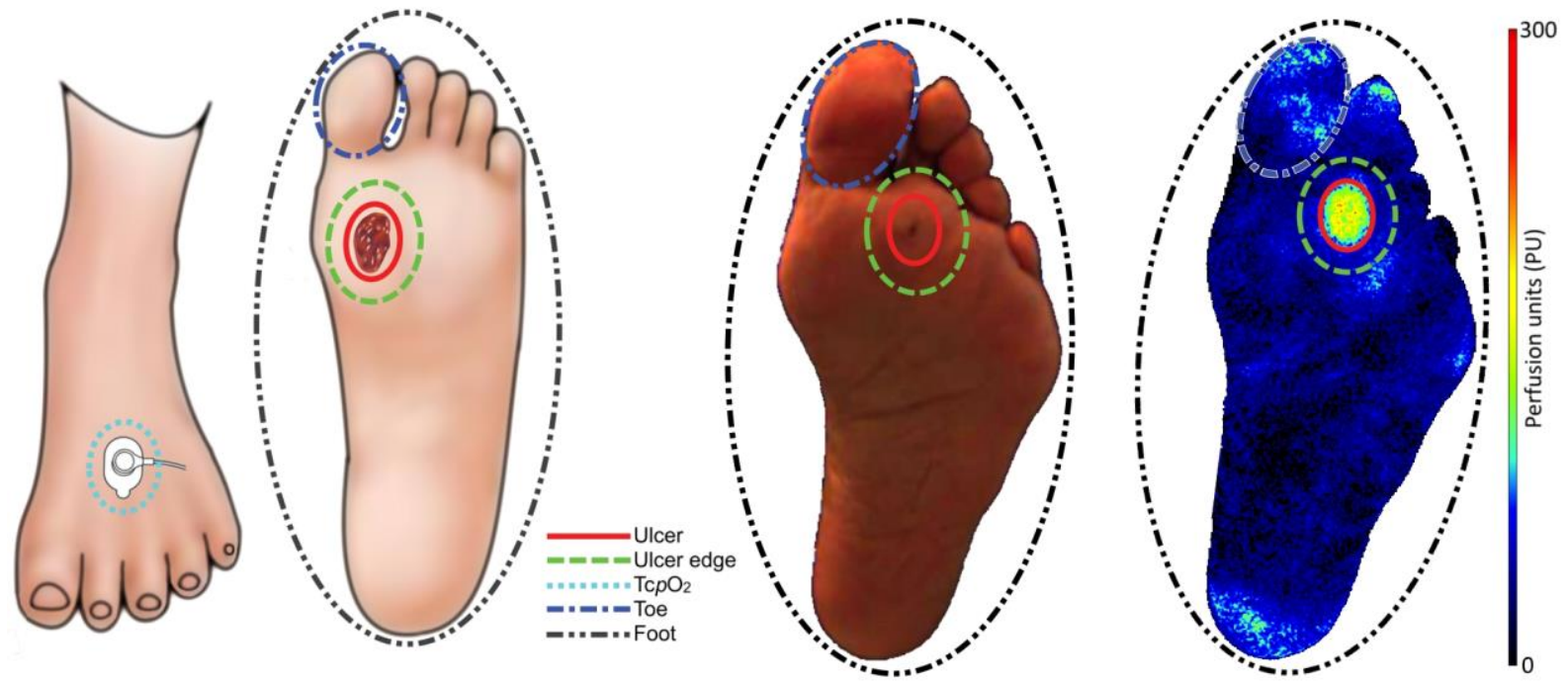

Fig. 2 Left: Schematic overview of the different Regions of Interest for perfusion measurements; ulcer location (edge) varied between participants. Middle and right: outcomes of an LSCI scan in one participant, with colour image (middle) and perfusion image (right).

Zero perfusion units from the background were removed from the picture and not used in the data analysis, because we excluded all zero-value pixels during calculations. The stability over time of the baseline measurements was analysed with linear mixed models. Intra- and interobserver correlations were calculated using intra-class correlations between and within different observers. Values $>0.7$ were considered high agreement (21). For the relation of LSCI and currently used non-invasive blood pressure measurements, Pearson's $r$ was calculated. A two-way random effect ANOVA was conducted to compare the inter-assessor reliability. Oneway ANOVA was performed to calculate the difference in microcirculation between the three patient populations (non-ischemic, ischemic and critical-ischemic), followed by post-hoc Bonferroni corrections for multiple comparisons between groups. With 3 conditions and 5 ROIs per patient, a total of 15 measurements were available for analyses. The relation between baseline measurement and post-occlusive peak or elevation test measurements was calculated with a Pearson's r. Alpha was set at $\leq 0.05$. Analyses were performed using SPSS version 21.0 (SPSS Inc. Chicago, IL, USA) and Microsoft Excel 2016 (Microsoft Corporation, Redmond, WA, USA). 


\section{Results}

The majority of the 33 included participants were males, mean age was around $64.9 \pm 13.6$ years, and most were diagnosed with type 2 diabetes (Table 1).

Table 1 Participant characteristics $(\mathrm{N}=33)$

\begin{tabular}{|c|c|c|}
\hline Characteristics & $\mathbf{N}$ & $\%$ \\
\hline \multicolumn{3}{|l|}{ Gender } \\
\hline Male & 28 & 85 \\
\hline Female & 5 & 15 \\
\hline \multicolumn{3}{|l|}{ Age (years) } \\
\hline mean $\pm \mathrm{SD}$ (range) & 64. & \\
\hline \multicolumn{3}{|l|}{ Diabetes Mellitus } \\
\hline Type 1 & 2 & 6 \\
\hline Type 2 & 31 & 94 \\
\hline \multicolumn{3}{|l|}{ Ulcer location } \\
\hline MTP1/dig 1 & 13 & 39 \\
\hline MTP2-3/dig 2-3 & 9 & 27 \\
\hline MTP4-5/dig 4-5 & 8 & 24 \\
\hline Other & 3 & 9 \\
\hline \multicolumn{3}{|l|}{ Ischemia } \\
\hline Non-ischemic & 11 & 33 \\
\hline Ischemic & 14 & 42 \\
\hline Critical-ischemic & 8 & 24 \\
\hline \multicolumn{3}{|l|}{ Neuropathic } \\
\hline Neuropathic impairment & 29 & 88 \\
\hline No neuropathic impairment & 4 & 12 \\
\hline
\end{tabular}

We found no significant differences between the three baselines over time (before stress tests 46.05 $\pm 13.15 \mathrm{PU}$, between stress tests $48.33 \pm 16.01 \mathrm{PU}$ and after stress tests $47.65 \pm 14.64 \mathrm{PU}$; $r=0.058)$. A high and significant intra-observer agreement was found for all 15 measurements ( $r=0.711-0.950 ; p \leq 0.001$; Table 2).

Table 2: Single measures Intra-class Correlation Coefficient of the microcirculation assessment with Laser Speckle Contrast Imaging in the foot for different regions of interest by: (A) one observer; (B) different observers and $(\mathrm{C})$ performed by secondary observer and analysed by the principal investigator and independent researcher

\begin{tabular}{|c|c|c|c|c|c|c|c|c|c|}
\hline \multirow[t]{2}{*}{ Characteristics } & \multicolumn{2}{|c|}{$\begin{array}{l}\text { Intra-observer } \\
\text { agreement }\end{array}$} & \multirow[b]{2}{*}{ 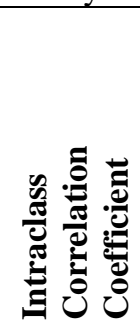 } & \multicolumn{2}{|c|}{$\begin{array}{l}\text { Inter-observer } \\
\text { agreement }\end{array}$} & \multirow[b]{2}{*}{ 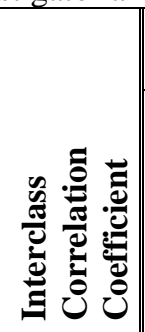 } & \multicolumn{2}{|c|}{$\begin{array}{l}\text { Inter-assessor } \\
\text { agreement }\end{array}$} & \multirow[b]{2}{*}{ 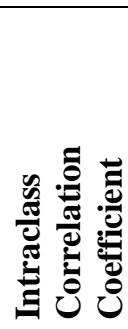 } \\
\hline & 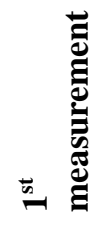 & స & & $\begin{array}{l}\overrightarrow{1} \\
\dot{0} \\
\dot{0} \\
0 \\
0\end{array}$ & 离 & & 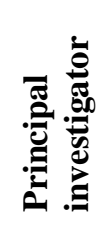 & 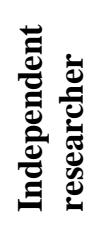 & \\
\hline \multicolumn{10}{|l|}{ Foot } \\
\hline Baseline & 46.05 & 46.76 & $0.950 *$ & 44.75 & 45.30 & $0.861 *$ & 45.30 & 45.50 & $0.990 *$ \\
\hline Post-occlusion peak & 82.24 & 80.79 & $0.761 *$ & 78.91 & 73.90 & $0.561 * *$ & 73.90 & 78.58 & $0.885^{*}$ \\
\hline
\end{tabular}




\begin{tabular}{|c|c|c|c|c|c|c|c|c|c|}
\hline Elevation test & 55.03 & 56.87 & $0.830^{*}$ & 53.83 & 54.69 & $0.825^{*}$ & 54.69 & 54.33 & $0.979 *$ \\
\hline \multicolumn{10}{|l|}{ Ulcer } \\
\hline Baseline & 103.54 & 101.57 & $0.758 *$ & 106.05 & 115.56 & $0.570 * *$ & 115.56 & 116.24 & $0.956 *$ \\
\hline Post-occlusion peak & 102.03 & 102.14 & $0.750 *$ & 104.37 & 112.24 & $0.562 * *$ & 112.24 & 113.10 & $0.897 *$ \\
\hline Elevation test & 101.34 & 104.50 & $0.711 *$ & 104.90 & 115.72 & $0.476 * *$ & 115.72 & 108.93 & $0.857^{*}$ \\
\hline \multicolumn{10}{|l|}{ Ulcer Edge } \\
\hline Baseline & 91.54 & 94.65 & $0.900 *$ & 93.48 & 98.25 & $0.783 *$ & 98.25 & 99.64 & $0.962 *$ \\
\hline Post-occlusion peak & 103.94 & 102.53 & $0.774 *$ & 104.31 & 105.67 & $0.728 *$ & 105.67 & 109.74 & $0.946 *$ \\
\hline Elevation test & 95.73 & 100.16 & $0.784 *$ & 98.28 & 103.17 & $0.518 * *$ & 103.17 & 103.28 & $0.942 *$ \\
\hline \multicolumn{10}{|l|}{ Toe } \\
\hline Baseline & 56.81 & 57.83 & $0.953 *$ & 52.33 & 54.92 & $0.858 *$ & 54.92 & 54.64 & $0.996 *$ \\
\hline Post-occlusion peak & 88.71 & 90.55 & $0.753 *$ & 86.41 & 84.17 & $0.714 *$ & 84.17 & 89.07 & $0.901 *$ \\
\hline Elevation test & 65.14 & 67.88 & $0.936 *$ & 63.52 & 66.01 & $0.818 *$ & 66.01 & 62.04 & $0.911 *$ \\
\hline \multicolumn{10}{|l|}{ ТcpO2 } \\
\hline Baseline & 16.03 & 12.68 & $0.879 *$ & 18.78 & 13.56 & $0.820 *$ & 42.38 & 42.12 & $0.992 *$ \\
\hline Post-occlusion peak & 25.00 & 21.74 & $0.827 *$ & 30.16 & 23.21 & $0.752 *$ & 72.52 & 78.10 & $0.900 * *$ \\
\hline Elevation test & 18.00 & 15.22 & $0.894 *$ & 22.02 & 18.36 & $0.877 *$ & 57.36 & 55.53 & $0.985^{*}$ \\
\hline
\end{tabular}

We found a high and significant inter-observer agreement for 10 out of 15 measurements (ICC $=0.728-0.861 ; p \leq 0.001)$, and a moderate and significant inter-observer agreement for the remaining 5 measurements (ICC $=0.476-0.570 ; p \leq 0.005$; Table 2 ). The inter-assessor agreement was high and significant (ICC $=0.857-0.996 ; p \leq 0.001$; Table 2). Low correlations were found between LSCI and ABI, toe pressure and $\mathrm{Tc} p \mathrm{O}_{2}$ (range -0.272-0.582; Table 3).

Table 3: Pearson's R correlations between Laser Speckle Contrast Imaging (LSCI) and non-invasive blood pressure measurements of the ulcerated foot for different Regions of Interest (ROI)

\begin{tabular}{|c|c|c|c|c|}
\hline ROI & Arm & Ankle & Toe & $\mathrm{TcpO} \mathrm{O}_{2}$ \\
\hline \multicolumn{5}{|l|}{ Foot } \\
\hline LSCI baseline & -0.272 & 0.112 & -0.048 & -0.066 \\
\hline Post-occlusion peak & -0.162 & 0.202 & $0.382 *$ & 0.110 \\
\hline Elevation test & -0.018 & $0.477 *$ & $0.488^{*}$ & 0.180 \\
\hline \multicolumn{5}{|l|}{ Ulcer } \\
\hline LSCI baseline & -0.154 & -0.166 & 0.078 & 0.036 \\
\hline Post-occlusion peak & -0.047 & 0.048 & 0.227 & 0.070 \\
\hline Elevation test & 0.018 & 0.228 & $0.429 *$ & 0.245 \\
\hline \multicolumn{5}{|l|}{ Ulcer edge } \\
\hline LSCI baseline & -0.228 & 0.062 & 0.127 & -0.007 \\
\hline Post-occlusion peak & -0.188 & 0.238 & $0.390 *$ & 0.245 \\
\hline Elevation test & -0.062 & $0.367 *$ & $0.533^{*}$ & 0.216 \\
\hline \multicolumn{5}{|l|}{ Toe } \\
\hline LSCI baseline & & & 0.233 & \\
\hline Post-occlusion peak & & & $0.500 *$ & \\
\hline Elevation test & & & $0.582 *$ & \\
\hline
\end{tabular}


$\mathrm{TcpO} \mathrm{O}_{2}$

LSCI baseline

0.062

Post-occlusion peak

0.232

Elevation test

0.242

Note: ${ }^{*} \mathrm{p} \leq 0.05$ (two-tailed)

No significant differences in perfusion between the critical ischemic, ischemic and nonischemic populations were found during the LSCI baseline measurements (Table 4; Fig. 3).

Table 4: ANOVA measurement with post hoc Bonferroni analysis for all participants with and without (critical-) ischemia for foot, hallux, ulcer and ulcer edge region of interest.

\begin{tabular}{|c|c|c|c|c|c|}
\hline & & $\begin{array}{l}\text { LSCI values } \\
\text { Perfusion units } \\
\text { (mean } \pm \text { SD) }\end{array}$ & $\begin{array}{l}\text { ANOVA } \\
p \text { value }\end{array}$ & $\begin{array}{l}\text { Post ho } \\
p \text { value }\end{array}$ & analysis \\
\hline Foot & & & & & \\
\hline LSCI baseline & Non-ischemic & $48.1 \pm 14.8$ & & NI - I & 1.000 \\
\hline & Ischemic & $46.9 \pm 12.7$ & 0.575 & $\mathrm{NI}-\mathrm{CI}$ & 0.947 \\
\hline & Critical-ischemic & $41.8 \pm 12.2$ & & I - CI & 1.000 \\
\hline Post-occlusion peak & Non-ischemic & $96.3 \pm 29.3$ & & NI - I & 0.284 \\
\hline & Ischemic & $79.6 \pm 18.4$ & $0.044^{*}$ & $\mathrm{NI}-\mathrm{CI}$ & $0.045^{*}$ \\
\hline & Critical-ischemic & $67.5 \pm 24.7$ & & I - CI & 0.791 \\
\hline Elevation test & Non-ischemic & $63.9 \pm 16.2$ & & NI - I & 0.468 \\
\hline & Ischemic & $56.1 \pm 10.0$ & $0.003 * *$ & $\mathrm{NI}-\mathrm{CI}$ & $0.003 * *$ \\
\hline & Critical-ischemic & $41.0 \pm 14.1$ & & I - CI & $0.046^{*}$ \\
\hline Toe & & & & & \\
\hline LSCI baseline & Non-ischemic & $68.0 \pm 28.9$ & & NI - I & 0.412 \\
\hline & Ischemic & $54.7 \pm 19.0$ & 0.081 & $\mathrm{NI}-\mathrm{CI}$ & 0.087 \\
\hline & Critical-ischemic & $45.0 \pm 11.8$ & & $\mathrm{I}-\mathrm{CI}$ & 0.953 \\
\hline Post-occlusion peak & Non-ischemic & $107.4 \pm 29.3$ & & NI - I & 0.167 \\
\hline & Ischemic & $86.7 \pm 20.2$ & $0.007 * *$ & $\mathrm{NI}-\mathrm{CI}$ & $0.006^{* *}$ \\
\hline & Critical-ischemic & $66.7 \pm 29.6$ & & $\mathrm{I}-\mathrm{CI}$ & 0.268 \\
\hline Elevation test & Non-ischemic & $83.0 \pm 28.7$ & & NI - I & 0.196 \\
\hline & Ischemic & $65.6 \pm 21.6$ & $0.001 * * *$ & $\mathrm{NI}-\mathrm{CI}$ & $0.001 * * *$ \\
\hline & Critical-ischemic & $39.9 \pm 12.5$ & & $\mathrm{I}-\mathrm{CI}$ & $0.047^{*}$ \\
\hline Ulcer & & & & & \\
\hline LSCI baseline & Non-ischemic & $113.4 \pm 23.7$ & & NI - I & 0.490 \\
\hline & Ischemic & $96.3 \pm 28.9$ & 0.370 & $\mathrm{NI}-\mathrm{CI}$ & 1.000 \\
\hline & Critical-ischemic & $102.6 \pm 38.0$ & & $\mathrm{I}-\mathrm{CI}$ & 1.000 \\
\hline Post-occlusion peak & Non-ischemic & $114.4 \pm 18.6$ & & NI - I & 0.105 \\
\hline & Ischemic & $92.7 \pm 27.2$ & 0.104 & $\mathrm{NI}-\mathrm{CI}$ & 0.784 \\
\hline & Critical-ischemic & $101.4 \pm 26.2$ & & I - CI & 1.000 \\
\hline Elevation test & Non-ischemic & $123.0 \pm 17.5$ & & NI - I & 0.053 \\
\hline & Ischemic & $96.1 \pm 27.1$ & $0.005^{* *}$ & $\mathrm{NI}-\mathrm{CI}$ & $0.005^{* *}$ \\
\hline & Critical-ischemic & $80.7 \pm 35.1$ & & I - CI & 0.604 \\
\hline Ulcer edge & & & & & \\
\hline LSCI baseline & Non-ischemic & $102.8 \pm 27.5$ & & NI - I & 0.396 \\
\hline & Ischemic & $87.5 \pm 19.1$ & 0.181 & $\mathrm{NI}-\mathrm{CI}$ & 0.288 \\
\hline & Critical-ischemic & $83.2 \pm 28.7$ & & I - CI & 1.000 \\
\hline Post-occlusion peak & Non-ischemic & $123.0 \pm 26.5$ & ด 006** & NI - I & $0.016^{*}$ \\
\hline & Ischemic & $96.1 \pm 21.5$ & $0.006^{*-n}$ & $\mathrm{NI}-\mathrm{CI}$ & $0.014 *$ \\
\hline
\end{tabular}




\begin{tabular}{|c|c|c|c|c|c|}
\hline & & & & \multirow{2}{*}{$\begin{array}{l}\text { I - CI } \\
\text { NI - I }\end{array}$} & \multirow{2}{*}{1.000} \\
\hline \multirow{2}{*}{ Elevation test } & Critical-ischemic & $91.5 \pm 15.8$ & \multirow{3}{*}{$\leq 0.001 * * *$} & & \\
\hline & Ischemic & $93.8 \pm 19.6$ & & $\mathrm{NI}-\mathrm{CI}$ & $\leq 0.001 * * *$ \\
\hline & Critical-ischemic & $70.8 \pm 23.6$ & & I - CI & 0.062 \\
\hline
\end{tabular}

Note: NI - I = Non-ischemic compared to Ischemic, NI - CI = Non-ischemic compared to Critical-ischemic, I - CI = Ischemic compared to Critical-ischemic; ${ }^{*} \mathrm{p} \leq 0.05, * * \mathrm{p} \leq 0.01,{ }^{* * *} \mathrm{p} \leq 0.001$
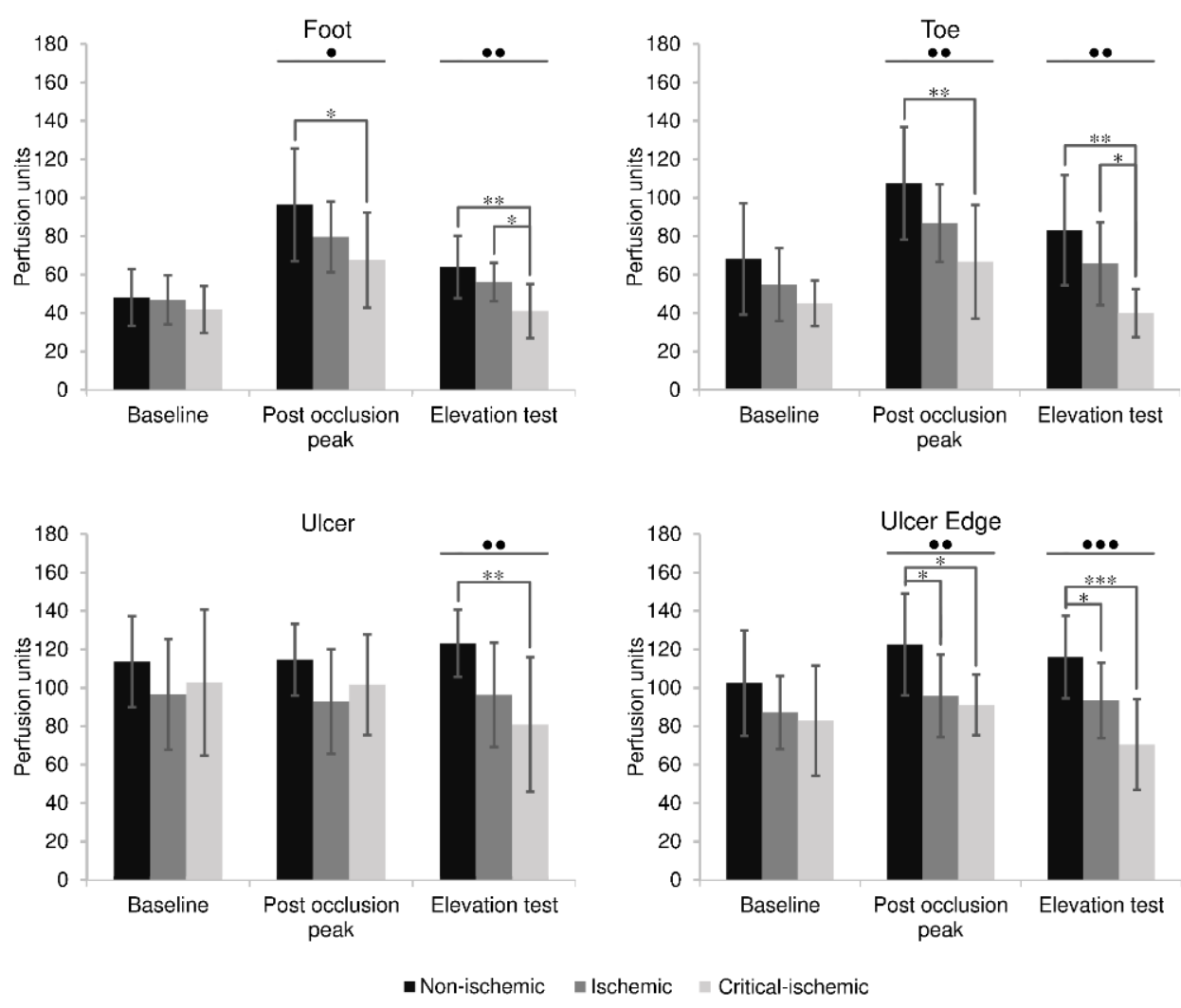

Fig. 3: LSCI values (perfusion units, mean \pm SD) of all participants with and without (critical-)ischemia for foot, toe, ulcer and ulcer edge ROIs.

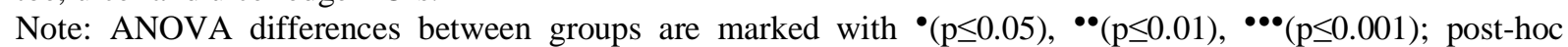
Bonferroni significant differences between groups are marked with $*(\mathrm{p} \leq 0.05), * *(\mathrm{p} \leq 0.01), * * *(\mathrm{p} \leq 0.001)$.

The perfusion during both stress tests was significantly higher for the non-ischemic population in comparison with the critical-ischemic population (Table 4; Fig. 3). The only exception was the post-occlusion peak for the ulcer ROI, which did not differ between populations. Further, perfusion was significantly higher in the ischemic vs. the critical-ischemic populations for the foot and toe ROIs during the elevation test, and significantly higher for the non-ischemic vs. the ischemic populations in the ulcer edge ROI during both stress tests, (Table 4; Fig. 3).

Moderate to good correlations (range $0.630-0.839 ; p \leq 0.001$ ) were found between the individual baseline measurements and the post-occlusive peak and elevating test values. Correlations for the post-occlusion peak were lower compared to elevation test values for all ROIs (Foot: 0.649 vs 0.779 ; Toe: 0.680 vs 0.833 ; Ulcer: 0.792 vs 0.798 ; Ulcer edge: 0.630 vs 
0.839). Correlations between baseline and stress test values for the different patient populations (non-ischemic, ischemic and critical-ischemic) show similar results (range 0.381-0.941), where post-occlusion peak were lower compared to elevation test values for all measurements except the toe values in the critical ischemic group (Fig. 4).

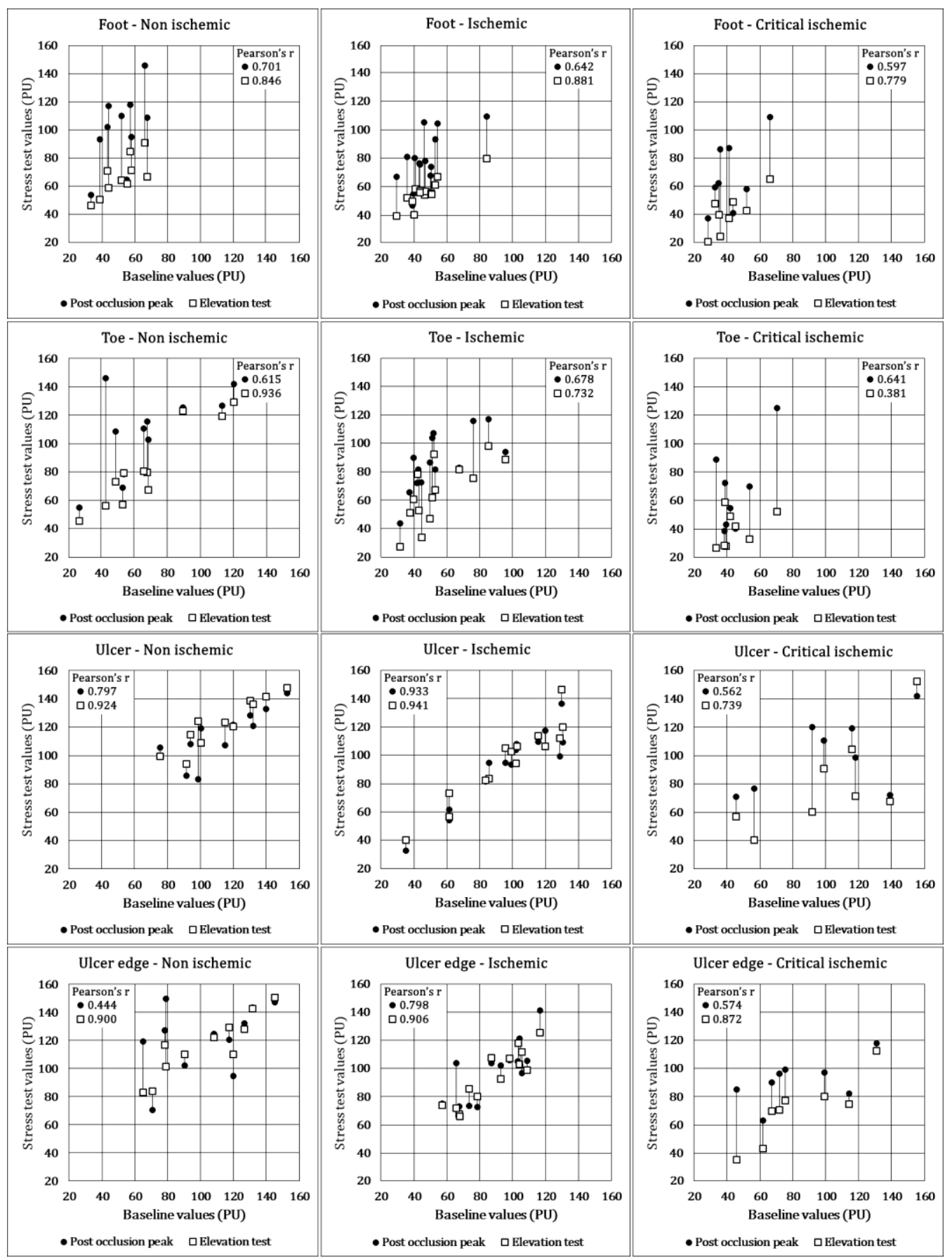

Fig. 4: LSCI baseline values compared to stress test values (post occlusion peak and Elevation test) of all participants with and without (critical-)ischemia for the foot, toe, ulcer and ulcer edge ROIs. Note: Vertical lines connect corresponding measurements of the same patients. 


\section{Discussion}

In this prospective cohort study, we investigated the reproducibility and stability of LSCI for the assessment of microcirculation in people with diabetic foot ulcers, and its relation with currently used non-invasive blood pressure measurements. We found high intra-observer agreements, moderate-to-high inter-observer agreements and high inter-assessor agreements; low correlations between LSCI and currently used non-invasive blood pressure measurements. LSCI was able to differentiate between non-ischemic, ischemic and critical-ischemic patients, especially during elevated blanching. LSCI can be regarded as a suitable technique to assess microcirculation in people with diabetic foot ulcers: it is stable and reproducible, non-invasive and non-contact, it does not disturb the blood flow, nor causes it damage to the tissues, and it can easily be implemented in routine outpatient clinical practice $(17,18,22)$.

Our findings regarding the intra-observer agreement are in line with other studies on LSCI for microcirculation assessment in other clinical populations, such as patients with clinical signs of peripheral arterial disease (23) and healthy populations $(24,25)$. In the study measured on peripheral arterial disease, perfusion was measured using LSCI in 63 feet of patients (23). They investigated reproducibility of 8 measurements, and found no significant differences between the measurements. Furthermore, the reproducibility of a post-occlusive peak test was measured for LSCI when measured on different days on the forearm of 28 healthy volunteers, and was found to be high (25). Finally, in a study on 10 subjects similar results were found compared to our work concerning performance of the post-occlusive peak test with LSCI, as they also found moderate-to-high inter-observer and intra-observer reproducibility of baseline, peak and plateaus (26).

This study was the first to also investigate inter-assessor agreement. We found high correlations, indicating that the influence of different assessors on the ROI placement or the removal of movement artefacts in the results is limited. This implies that data extraction from scans can be safely done by different technicians. However, it is still a useful avenue for future research to automate this step, as the current analyses are time-consuming and thereby a barrier for effective clinical implementation.

The low correlation between LSCI and conventional blood pressure measurements was anticipated, and in line with the aforementioned clinical population of 63 peripheral arterial disease patients (23). We confirm their conclusion that baseline perfusion values of LSCI are not correlated with non-invasive blood pressure measurements (e.g. ABI and tcPO2). This suggests that LSCI measurements differ from currently used non-invasive blood pressure measurements. This is likely the result of measuring at different area and depth with LSCI in 
comparison to non-invasive blood pressure measurement. For example, toe and ankle pressure (and related indices) or $\mathrm{Tc} p \mathrm{O}_{2}$ do not necessarily reflect the microcirculatory flow at the ulcer site, the ulcer edge or the whole foot. With LSCI, the whole foot can be measured, as well as individual ROIs. The penetration depth of LSCI is approximately $0.3 \mathrm{~mm}$ (26), measuring circulation predominantly in the skin arterioles, whereas non-invasive blood pressure is derived from arteries. Although non-invasive blood pressure measurements are the current standard for initial prediction of diabetic foot ulcer healing tendency (2), we expect that with the measurement of microcirculation in the arterioles closer to the ulcer, a better indication might be available of the situation at or around the ulcer. This might lead to an improvement of the moderate predictions of the healing tendency currently achieved (9).

A requirement to improve this healing tendency prediction, is that LSCI should discriminate between critical-ischemic, ischemic and non-ischemic patients. Although we found different perfusion values for each patient population, with non-ischemic patients having the highest perfusion values and critical-ischemic the lowest, the differences were not significant during the baseline measurements or at the ulcer ROI. Both stress tests enlarged these differences and reached statistical significance in most ROIs. This indicates that potential usefulness of LSCI lies especially in combination with those stress tests. The increased perfusion value during the elevated blanching tests could be explained by the veno-arteriolar reflex causing vasoconstriction of the microvascular bed when the foot is lowered (27). It could be possible that the lifting of the leg causes a vasodilatation and increases the perfusion values and differences between the non-ischemic and critical-ischemic patient population during the stress test.

Differences between the three populations were smaller at the ulcer ROI than at the ulcer edge ROI. This could be explained by the angiogenesis and reepithelialisation that form from the ulcer edge towards the centre of the ulcer area, and with more microcirculatory activity in non-ischemic patients compared to the (critical-)ischemic patient population. The difference in LSCI at the ulcer location between non-ischemic and (critical-)ischemic patients was smaller. After debridement it is possible that fluid, nutrients, and immune-competent cells are transported to the ulcer and increase the LSCI signal (28), and all our participants were measured after debridement. Other studies have shown similar findings, for example when microcirculation in and around diabetic foot ulcers was measured with hyperspectral imaging $(29,30)$. They found a significant negative correlation between the hyperspectral imaging signal in intact skin adjacent to the edge of the ulcer and ulcer healing (30). We expect that LSCI may complement non-invasive blood pressure measurement, to assess the microcirculation in this 
population. Based on the current results, we suggest that microcirculation in the ulcer edge could be the best indicator, and application of different stress test during the measurements is needed.

Different optical imaging techniques are also available to monitor microcirculation in diabetic foot ulcers. For example Laser Doppler Perfusion Imaging (LDPI), Hyperspectral Imaging (HSI) and Photoacoustic Imaging (31). Each technique has their own advantages and disadvantages and can be used to measure different characteristics of the microcirculation in tissue or the diabetic foot (31). The non-contact and full field imaging possibilities of LSCI, LDPI and HSI are great advantages for clinical measurements. Furthermore, the compact design, low cost and short imaging time of the LSCI make it an interesting technique to measure perfusion in the diabetic foot and to investigate the feasibility of LSCI in a clinical setting.

This study has limitations. First, the differentiation between the three patient populations was based on non-invasive blood pressure measurements. This is in line with international guidelines (2), but still includes the limitations of these non-invasive tests as discussed above. Unfortunately, no other non-invasive criteria are currently available. The use of invasive or extensive diagnostic techniques, like angiography or duplex, was not desirable in this study. We did not want to expose participants to invasive tests when these were not needed for their regular treatment. A potential effect of this limitation is dividing participants into the wrong category of ischemia, while this could not be checked with invasive diagnostics. This could result in a different distribution of the patient populations and may influence the results, as a different distribution of patients based on other diagnostic techniques would result in different mean LSCI values for each patient population.

Another limitation was the relatively small study size, and the not entirely equal distribution of the number of the non-ischemic, ischemic and critical-ischemic participants. However, our total of 33 participants was sufficient to obtain first insights in the use of LSCI, and the number of participants is comparable to related studies $(23,30)$. With the variations seen between patients with diabetic foot ulcers, assessment of LSCI in larger populations is needed in future research (11). Furthermore, no drug related medical background of the patients was included in this study. Therefore it was not possible to monitor and measure the potential effects of any vasoactive drugs on the measured perfusion values. This could be a confounding factor in our results.

We did not measure the temperature of the foot during each measurement. However, the LSCI scans were performed in a temperature controlled hospital room $\left(21 \pm 1^{\circ} \mathrm{C}\right)$ end the foot 
had sufficient time to acclimatise to this room temperature, but we cannot correct for possible differences in skin temperate between individual patients.

Another limitation was the neuropathic status of the patients. We measured the neurological impairment of all patients based on loss of protective sensation and were not able to perform a statistical analysis due to the small patient population without neurological impairment. Furthermore, we think that a better scaling and measurement of the degree of neurological impairment could be useful to show a better correlation between measured perfusion and neuropathic status. For example, a quantitative scaling method could give more insight in the correlation between measured perfusion and neurological impairment instead of measuring only the presence or absence of neurological impairment as we did in the current study.

This study provides first insights in the potential of LSCI in the assessment of microcirculation in the diabetic foot. Future research should examine the added diagnostic value of LSCI in clinical practice. As the aim of this study was to investigate the feasibility of LSCI, we did not investigate clinical outcomes in the current study. For this, a larger cohort of patients is needed that is followed over time, to link microcirculation assessment with clinical outcomes The ability of LSCI to show significant differences between different patient groups indicates that threshold baseline LSCI-values to predict wound healing might be derived from such future research and could be used to predict healing outcome and help with diagnoses and the choice in adequate treatment options.

\section{Conclusion}

LSCI is a stable and reproducible technique for the assessment of microcirculation in people with diabetic foot ulcers and its result using various stress tests show significant differences between non-ischemic, ischemic and critical-ischemic patient populations. LSCI provides a new assessment method for microcirculation in this population and may complement noninvasive blood pressure techniques. 


\section{References}

1. Bakker K, Apelqvist J, Lipsky B, Van Netten J, International Working Group on the Diabetic foot. The 2015 IWGDF Guidance documents on prevention and management of foot problems in diabetes: development of an evidence-based global consensus. Diabetes Metab Res Rev. 2016;32:2-6.

2. Hinchliffe R, Brownrigg J, Apelqvist J, Boyko EJ, Fitridge R, Mills J, et al. IWGDF guidance on the diagnosis, prognosis and management of peripheral artery disease in patients with foot ulcers in diabetes. Diabetes Metab Res Rev. 2016;32:37-44.

3. Aldworth J, Bache N Al, Hegelund MH, Hirst SM, Linnenkamp U, Magliano D, et al. IDF Diabetes Atlas 7th edition. 2015. 144 p.

4. Armstrong DG, Boulton AJM, Bus SA. Diabetic Foot Ulcers and Their Recurrence. N Engl J Med. 2017;376(24):2367-75.

5. Alexiadou K, Doupis J. Management of diabetic foot ulcers. Diabetes Ther. 2012;3(1):1-15.

6. Kerr M, Rayman G, Jeffcoate WJ. Cost of diabetic foot disease to the National Health Service in England. Diabet Med. 2014 Dec;31(12):1498-504.

7. Prompers L, Huijberts M, Schaper N, Apelqvist J, Bakker K, Edmonds M, et al. Resource utilisation and costs associated with the treatment of diabetic foot ulcers. Prospective data from the Eurodiale Study. Diabetologia. 2008;51(10):1826-34.

8. Prompers L, Schaper N, Apelqvist J, Edmonds M, Jude E, Mauricio D, et al. Prediction of outcome in individuals with diabetic foot ulcers: Focus on the differences between individuals with and without peripheral arterial disease. The EURODIALE Study. Diabetologia. 2008;51(5):747-55.

9. Brownrigg JRWW, Hinchliffe RJ, Apelqvist J, Boyko EJ, Fitridge R, Mills JL, et al. Performance of prognostic markers in the prediction of wound healing or amputation among patients with foot ulcers in diabetes: A systematic review. Diabetes Metab Res Rev. 2016;32:128-35.

10. Sumpio BE, Forsythe RO, Ziegler KR, van Baal JG, Lepantalo MJAA, Hinchliffe RJ. Clinical implications of the angiosome model in peripheral vascular disease. J Vasc Surg. 2013;58(3):814-26.

11. Elgzyri T, Larsson J, Thörne J, Eriksson K-FF, Apelqvist J. Outcome of Ischemic Foot Ulcer in Diabetic Patients Who Had no Invasive Vascular Intervention. Eur J Vasc Endovasc Surg. 2013;46(1):110-7.

12. Draijer M, Hondebrink E, Van Leeuwen T, Steenbergen W. Review of laser speckle contrast techniques for visualizing tissue perfusion. Lasers Med Sci. 2009;24(4):639-51.

13. Boas DA, Dunn AK. Laser speckle contrast imaging in biomedical optics. J Biomed Opt. 2010;15(1):011109.

14. Biffi CA, Tuissi A. Stato dell'arte sulle tecniche di produzione additiva per metalli. Metall Ital. 
2017;109(1):5-10.

15. Li H, Liu Q, Lu H, Li Y, Zhang HF, Tong S. Directly measuring absolute flow speed by frequency-domain laser speckle imaging. Vol. 22, Optics express. 2010. 21079 p.

16. Ganapathy P, Tamminedi T, Qin Y, Nanney L, Cardwell N, Pollins A, et al. Dual-imaging system for burn depth diagnosis. Burns. 2014;40(1):67-81.

17. de M. Matheus AS, Clemente ELS, de Lourdes Guimarães Rodrigues M, Torres Valença DC, Gomes MB. Assessment of microvascular endothelial function in type 1 diabetes using laser speckle contrast imaging. J Diabetes Complications. 2017;31(4):753-7.

18. Jayanthy AK, Sujatha N, Reddy MR, Narayanamoorthy VB. Non invasive blood flow assessment in diabetic foot ulcer using laser speckle contrast imaging technique. In: Wax A, Backman V, editors. Arch Ophthalmol. International Society for Optics and Photonics; 2014. p. 89521D.

19. Lipsky BA, Aragón-Sánchez J, Diggle M, Embil J, Kono S, Lavery L, et al. IWGDF guidance on the diagnosis and management of foot infections in persons with diabetes. Diabetes Metab Res Rev. 2016;32:45-74.

20. Spångeus A, Wijkman M, Lindström T, Engvall JE, Östgren CJ, Spångéus A, et al. Toe brachial index in middle aged patients with diabetes mellitus type 2: Not just a peripheral issue. Diabetes Res Clin Pract. 2013;100(2):195-202.

21. Landis JR, Koch GG. The Measurement of Observer Agreement for Categorical Data. Biometrics. 1977;33(1):159.

22. Allen J, Howell K. Microvascular imaging: techniques and opportunities for clinical physiological measurements. Physiol Meas. 2014;35(7):R91-141.

23. Katsui S, Inoue Y, Igari K, Toyofuku T, Kudo T, Uetake H. Novel assessment tool based on laser speckle contrast imaging to diagnose severe ischemia in the lower limb for patients with peripheral arterial disease. Lasers Surg Med. 2017;49(7):645-51.

24. Humeau-Heurtier A, Abraham P, Durand S, Mahé G. Excellent inter- and intra-observer reproducibility of microvascular tests using laser speckle contrast imaging. Clin Hemorheol Microcirc. 2013;58(3):439-46.

25. Roustit M, Millet C, Blaise S, Dufournet B, Cracowski JL. Excellent reproducibility of laser speckle contrast imaging to assess skin microvascular reactivity. Microvasc Res. 2010;80(3):505-11.

26. O’Doherty J, McNamara P, Clancy NT, Enfield JG, Leahy MJ. Comparison of instruments for investigation of microcirculatory blood flow and red blood cell concentration. J Biomed Opt. 2009;14(3):034025.

27. Rayman G, Hassan A, Tooke JE. Blood flow in the skin of the foot related to posture in diabetes mellitus. Br Med J (Clin Res Ed). 1986 Jan 11;292(6513):87-90.

28. Gonzalez AC de O, Costa TF, Andrade Z de A, Medrado ARAP. Wound healing - A literature 
review. An Bras Dermatol. 2016;91(5):614-20.

29. Yudovsky D, Nouvong A, Pilon L. Hyperspectral imaging in diabetic foot wound care. J Diabetes Sci Technol. 2010 Sep;4(5):1099-113.

30. Jeffcoate WJ, Clark DJ, Savic N, Rodmell PI, Hinchliffe RJ, Musgrove A, et al. Use of HSI to measure oxygen saturation in the lower limb and its correlation with healing of foot ulcers in diabetes. Diabet Med. 2015;32(6):798-802.

31. Mennes OA, van Netten JJ, Slart RHJ., Steenbergen W. Novel Optical Techniques for Imaging Microcirculation in the Diabetic Foot. Curr Pharm Des. 2018;24(12):1304-16. 